\title{
CHOLECYSTOCUTANEOUS FISTULA
}

\section{GONDIL $S^{1}$, KANASE $V^{2}$ AND KAUSHIK $T^{3}$}

1: Junior Resident, Department of Surgery, Krishna Institute of Medical Science Deemed to be University, Karad Maharashtra (India)

2: Professor, Department of Surgery, Krishna Institute of Medical Science Deemed to be University, Karad Maharashtra (India)

3: Senior Resident, Department of Surgery, Krishna Institute of Medical Science Deemed to be University, Karad Maharashtra (India)

*Corresponding Author: Dr. Saurabh Gondil: E Mail: gsaurabh.gondil@gmail.com Received 12 ${ }^{\text {th }}$ July 2021; Revised 14 ${ }^{\text {th }}$ Aug. 2021; Accepted $27^{\text {th }}$ Oct. 2021; Available online $15^{\text {th }}$ Feb. 2022 https://doi.org/10.31032/IJBPAS/2022/11.2.1012

\section{INTRODUCTION:}

An abnormal connection between two epithelial surfaces is called a fistula. This abnormal communication can result from a complication of a disease or surgery. The name of the fistula follows the two surfaces or lumens that it connects [2]. A cholecystocutaneous fistula is an unusual connection between the gallbladder and the skin. The cholecystocutaneous fistula is a rare clinical entity in surgical practice today. There are reports that have recorded events of spontaneous cholecystocutaneous fistulas occurring as early as the 17th century. In the same way, an enterocutaneous fistula is an abnormal communication between the small intestine and the skin. The development of the disease results in complexity due to neglected calcium cholecystitis though, it happens extremely rare in today's surgical practice. As a result of improvements in diagnostic imaging, the bile duct diseases are being diagnosed early these days. The availability of effective and safe surgical treatment has made complications such as fistula extremely rare $[\mathbf{1 , 3}, \mathbf{6}]$.

\section{Etiology:}

Cholecystocutaneous fistulas are a rare complication of ignored calcium cholecystitis, which has also been associated with acalculous cholecystitis or the gallbladder carcinoma. When the 
gallbladder wall is aroused, it begins to necrosis if left as it is. The entire wall necrosis causes bile to leak into adjoining tissues. The most familiar locations for bile leakage to be observed is the abdominal wall and possibly the covering skin causing the cholecystocutaneous fistula [5].

\section{Epidemiology:}

The concerned patients are generally older women. Because of the disease being rare, the information available about it, is minimal. While examining the patients they may cite previous events of pain observed in the right hypochondrium which implies to gallstone disease. fistula opening is most frequently sited in the top right quadrant of the abdomen. The painless and draining fistulas matching with this situation have been recorded in many places of the abdominal wall, such as the right upper quadrant, iliac fossa, umbilicus, lumbar region, and left lower quadrant, also up to the gluteal region. These fistulas are recognized to appear after percutaneous treatment for acute cholecystitis and recurrence of the cholecystitis alongside fistula development in the earlier pathway of cholecystostomy drain $[4,8]$.

\section{Pathophysiology:}

The hindrance in the bile flow plays an integral part in the pathophysiology of the expansion of the condition. Unrelieved obstruction of bile flow compromises blood flow to the gallbladder wall and lymphatic drainage, which ultimately results in necrosis of the gallbladder wall and hence blockage of the cystic duct advances to an increase in intravesicular pressure. The open perforation of the pericholecystic gallbladder abscess ensues. Once punctured, the gallbladder can drain into the peritoneal cavity, causing a localized Perito-ni abscess which can then develop into an external fistula because of its adhesion to the abdominal wall. It can expel its contents through an area of least resistance or the shortest path. Gallbladder carcinoma is known to cause an external fistula. Gallbladder skin fistula is usually a late sequel to chronic bile duct disease, which has been reported after inadequate treatment for acute cholecystitis.

\section{History and Physical:}

The typical clinical presentation is a sinus that is persistently draining bile. In such cases, there is usually a history of chronic bile duct disease, although the patients do not report it as a primary complaint. Sometimes the patient may also have a history of gallstone expelling through the fistula. The most usual site of the external opening is in the upper right quadrant of the abdominal wall. However, the opening of the fistula path could be present in the left costal margin or the right (iliac fossa, groin, gluteal) region. Patients can be toxic with 
acute infection or related abscesses. The character of the fistula discharge may vary depending on the underlying pathology.

\section{Evaluation:}

USG is important in the background of gallstone disease, but it is not of significant use in gallbladder fistula even though it reliably demonstrates the presence of gallstones. Computed tomography (CT) scan is the most helpful imaging modality for diagnosing this complication. It will show the presence of gallstones and communication to the gallbladder. It also indicates the existence of any internal fistula and other associated pathology. MRI with MRCP has also been found to help establish the diagnosis $[\mathbf{7 , 9 ]}$.

\section{Treatment / Management:}

For cholecystic skin fistula drainage, appropriate antibiotics are necessary. If there is an associated mass, then it is recommended to obtain a cytologic diagnosis by fine needle cytology puncture (FNAC) to rule out the underlying root cause. Cytology being negative or in the case of no malignancy suspicion, action to be begun is an elective cholecystectomy only after the patient's state is stable with the general condition optimized. Open cholecystectomy along with excision of the fistulous tract is the permanent cure. Percutaneous treatment with gallstone removal is only reserved for older people with multiple corresponding co-morbidities or those who are too weakened to withstand surgery. Before definitive surgery, an effort should be made to ensure no obstruction of the bile ducts or associated stones. An unresectable disease is determined if a gallbladder fistula occurs as part of cancer with an associated dated mass $[\mathbf{1 1}, \mathbf{1 2}]$.

\section{Prognosis:}

The prognosis for cholecystocutaneous fistula is generally reasonable. Complications can appear in older people with underlying co-morbidities. Malignant changes in the fistula tract are rare.

\section{Complications:}

- Necrotizing fasciitis

- Skin irritation

- Malignant changes

Enhancing The Healthcare Team Outcomes:

Cholecysto-cutaneous fistulas are infrequent today, but like any skin fistula, they require adequate drainage and skin protection while managing the root cause. Cholecystocutaneous fistulas are generally handled by nurses on a daily basis in the same way as any skin fistula by recording the amount of drainage, characteristics of the drainage, and providing protection for the skin at the same time. If any abnormality occurs, such as slow drainage, pus, or signs of infection, the surgeon should be contacted straight away. The 
nurse must monitor the patient and provide support to the family, including ongoing education of the patient and their family. The prognosis for malignant is guarded, but the prognosis for benign is good. Healthcare workers need to know that untreated chronic wounds can develop malignant changes after 10 to 20 years. Close supervision by the inter-professional team is therefore necessary. The best results can be obtained by interprofessional teamwork in the treatment of these difficult cases $[7,9,10]$.

\section{Acknowledgment}

I am thankful to Rupali Salunkhe for secretarial help, Department of Surgery, Krishna Institute of Medical Science Deemed to be University, Karad Maharashtra (India)

\section{REFERENCES:-}

[1] Waheed A, Mathew G, Tuma F, Cholecystocutaneous Fistula, StatPearls [Internet], 2020.

[2] Shams C, Cannon M, Bortman J, Hakim S, Stone, Induced purulent choledocoduodenal fistula presenting with ascending cholangitis, ACG case reports journal, 5, 2018.

[3] Wang YF, Chen HQ. Hepatobiliary and Pancreatic: Spontaneous cholecystocutaneous fistula, Journal of gastroenterology and hepatology, 34(1), 2019, 11.
[4] Jayasinghe G, Adam J, Abdul-Aal Y, Unusual presentation of gallbladder perforation, International journal of surgery case reports, 18, 2016, 42-4.

[5] Gerrard A, Date R, Delayed Spontaneous Passage of Gallstones via Cholecystocutaneous Fistula, ACG case reports journal, 4, 2017.

[6] Rinzivillo NM, Danna R, Leanza V, Lodato M, Marchese S, Basile F, et al, Spontaneous cholecystocutaneous fistula, a rare cholelithiasis complication, F1000 Research, 6, 2017.

[7] Pripotnev S, Petrakos A, Cholecystocutaneous fistula after percutaneous gallbladder drainage, Case reports in gastroenterology, 8(1), 2014, 119-22.

[8] Maynard W, McGlone ER, Deguara J, Unusual aetiology of abdominal wall abscess: cholecystocutaneous fistula presenting 20 years after open subtotal cholecystectomy, Case Reports, 2016, bcr2015213326.

[9] Micu BV, Andercou OA, Micu CM, Militaru V, Jeican II, Bungărdean CI, et al, Spontaneous cholecystocutaneous fistula as a primary manifestation of gallbladder adenocarcinoma associated with gallbladder lithiasis-case report, Rom J Morphol Embryol, 58(2), 2017, $575-83$. 
[10] Ozdemir Y, Yucel E, Sucullu I, Filiz I,

Gulec B, Akin ML, et al, Spontaneous

cholecystocutaneous fistula as a rare complication of gallstones, Bratislavske lekarske listy, 113(7), 2012, 445-7.

[11]Kapoor Y, Singh G, Khokhar M, Spontaneous cholecystocutaneous fistula - not an old-time story, Indian Journal of Surgery, 75(1), 2013, 18891.

[12] Jayant M, Kaushik R, Attri AK, Spontaneous cholecystocutaneous abscess, Indian Journal of Gastroenterology, 33(5), 2014, 498. 